\title{
A SPECTROPOLARIMETRIC TEST OF THE STRUCTURE OF THE INTRINSIC ABSORBERS IN THE QUASAR
} HS $1603+3820^{*}$

\author{
Toru Misawa ${ }^{1,2,3}$, Koji S. Kawabata ${ }^{4}$, Michael Eracleous ${ }^{2,5}$, Jane C. Charlton ${ }^{2}$, and Nobunari Kashikawa ${ }^{6}$ \\ ${ }^{1}$ Cosmic Radiation Laboratory, RIKEN, 2-1 Hirosawa, Wako, Saitama 351-0198, Japan; misawatr@ shinshu-u.ac.jp \\ ${ }^{2}$ Department of Astronomy and Astrophysics, The Pennsylvania State University, University Park, PA 16802, USA; mce@ astro.psu.edu, charlton@astro.psu.edu \\ ${ }^{3}$ School of General Education, Shinshu University, 3-1-1 Asahi, Matsumoto, Nagano 390-8621, Japan \\ ${ }^{4}$ Hiroshima Astrophysical Science Center, Hiroshima University, 1-3-1 Kagamiyama, Higashi-Hiroshima, Hiroshima 739-8526, Japan; kawabtkj@ hiroshima-u.ac.jp \\ ${ }^{5}$ Center for Gravitational Wave Physics, The Pennsylvania State University, University Park, PA 16802, USA \\ ${ }^{6}$ National Astronomical Observatory, Mitaka, Tokyo 181-8588, Japan; kashik@zone.mtk.nao.ac.jp \\ Received 2009 November 17; accepted 2010 June 29; published 2010 August 4
}

\begin{abstract}
We report the results of a spectropolarimetric observation of the C VI "mini-broad" absorption line (mini-BAL) in the quasar HS $1603+3820\left(z_{\mathrm{em}}=2.542\right)$. The observations were carried out with the FOCAS instrument on the Subaru Telescope and yielded an extremely high polarization sensitivity of $\delta p \sim 0.1 \%$, at a resolving power of $R \sim 1500$. HS $1603+3820$ has been the target of a high-resolution spectroscopic monitoring campaign for more than four years, aimed at studying its highly variable C VI mini-BAL profile. Using the monitoring observations in an earlier paper, we were able to narrow down the causes of the variability to the following two scenarios: (1) scattering material of variable optical depth redirecting photons around the absorber and (2) a variable, highly ionized screen between the continuum source and the absorber which modulates the UV continuum incident on the absorber. The observations presented here provide a crucial test of the scattering scenario and lead us to disfavor it because (1) the polarization level is very small $(p \sim 0.6 \%)$ throughout the spectrum and (2) the polarization level does not increase across the mini-BAL trough. Thus, the variable screen scenario emerges as our favored explanation of the C vi mini-BAL variability. Our conclusion is bolstered by recent X-ray observations of nearby mini-BAL quasars, which show a rapidly variable soft X-ray continuum that appears to be the result of transmission through an ionized absorber of variable ionization parameter and optical depth.
\end{abstract}

Key words: quasars: absorption lines - quasars: individual (HS 1603+3820)

Online-only material: color figures

\section{INTRODUCTION}

Quasars are routinely used as background sources, allowing us to study intervening gaseous structures via absorption-line diagnostics. The observed absorption lines have their origin in both intervening objects, such as foreground galaxies, the intergalactic medium (IGM), or the interstellar medium (ISM) of the quasar host galaxies, and in structures that are intrinsic to the quasars.

A promising candidate for the intrinsic absorbers is an outflowing wind from the accretion disk that makes up the quasar central engine. The outflow is thought to be accelerated by either magnetocentrifugal forces (e.g., Everett 2005; de Kool \& Begelman 1995), by radiation pressure in lines and continuum (Murray et al. 1995; Proga et al. 2000), and/or by radiation pressure acting on dust (e.g., Voit et al. 1993; Königl \& Kartje 1994). According to many of the above models, the flow can be equatorial, although the opening angle relative to the plane of the disk depends on the choice of model parameters. In other models, the flow can be polar (directed at right angles to the disk plane; e.g., Königl \& Kartje 1994; Punsly 1999).

The outflowing winds are important in two ways. First, they may be essential components of the quasar central engine: they can carry angular momentum away from the disk, allowing accretion onto the black hole to proceed. Second, they can deliver energy and momentum to the ISM and IGM, thus regulating gas infall and star formation during the assembly

\footnotetext{
* Based on data collected at Subaru Telescope, which is operated by the National Astronomical Observatory of Japan.
}

of a galaxy (e.g., Granato et al. 2004; Scannapieco \& Oh 2004; Springel et al. 2005). Thus, feedback from quasar outflows can profoundly affect the properties of present-day galaxies and it can also enrich the IGM with metals. For these reasons, it is important to understand the properties and structure of quasar outflows, a goal which can be accomplished by studying the absorption lines that they produce.

\subsection{BALs, NALs, and Mini-BALs}

In the spectra of quasars, the broad absorption lines (BALs; FWHM $\geqslant 2000 \mathrm{~km} \mathrm{~s}^{-1}$ ) are easily identified as intrinsic. The large widths of BALs make them very unlikely to originate in intervening objects or the ISM of the quasar host galaxy. BALs are detected in $10 \%-20 \%$ of optically selected quasars (e.g., Hewett \& Foltz 2003; Reichard et al. 2003), and the detection rate is slightly higher in radio-quiet quasars (e.g., Stocke et al. 1992; Becker et al. 2001; Gregg et al. 2006). ${ }^{7}$ The observed fraction of quasars hosting BALs constrains the solid angle subtended by the dense portion of the wind to the central engine.

Narrow absorption lines (NALs) observed in the spectra of quasars (FWHM $\leqslant 500 \mathrm{~km} \mathrm{~s}^{-1}$ ) are also physically associated with the quasars. This was motivated by the fact that the detection rate of NALs varies between sight lines and is related to the physical properties of the background quasars (Richards et al. 1999; Richards 2001). Such a trend is not expected, if all NALs arise in intervening absorbers. This suggestion was

\footnotetext{
7 Chartas (2000) found that approximately $35 \%$ of radio-quiet gravitational lensed quasars contain BAL features, which suggest that flux-limited optical surveys may be underestimating the fraction of BALs.
} 
confirmed once NALs were completely de-blended into multiple narrower components with high-resolution spectroscopy. We can now separate intrinsic NALs from intervening ones via two characteristic properties related to the compactness of the gas parcels in intrinsic absorbers: (1) dilution of absorption troughs by un-occulted light from the background source(s) associated with the quasar (hereafter "partial coverage;" e.g., Barlow \& Sargent 1997; Hamann et al. 1997a; Ganguly et al. 1999) and (2) time variability of line profiles (e.g., depth, equivalent width, and line centroid), within $\lesssim 1 \mathrm{yr}$ in the quasar rest frame (e.g., Hamann et al. 1997b; Narayanan et al. 2004; Wise et al. 2004). Neither of these properties is expected in intervening absorbers because they have much larger sizes and lower densities compared to intrinsic absorbers. Using the former test, Misawa et al. (2007a) find that $~ 50 \%$ of non-BAL QSOs at $z=2-4$ have at least one intrinsic NAL in their restframe, near-UV spectra.

Equally useful, but less common, are mini-BALs, with intermediate line widths between NALs and BALs $\left(500 \mathrm{~km} \mathrm{~s}^{-1} \leqslant\right.$ FWHM $\leqslant 2000 \mathrm{~km} \mathrm{~s}^{-1}$ ). Mini-BALs are also identified by partial coverage and/or time variability. However, they can also be identified just from their smooth and relatively broad profiles, which are incompatible with the properties of intervening gaseous structures. Mini-BALs have the advantages of both BALs (i.e., high probability of being intrinsic) and NALs (i.e., line profiles can be resolved into individual components), which make them useful as diagnostics (e.g., Churchill et al. 1999; Hamann et al. 1997b; Narayanan et al. 2004).

BALs, mini-BALs, and NALs could be complementary probes of quasar outflows since they may be observed along different lines of sight toward the quasar continuum and/or emission-line source. This idea is bolstered by the results of X-ray observations by Misawa et al. (2008) and Chartas et al. (2009), which show that the NAL velocities and the column densities of the corresponding X-ray absorbers follow a different relation from BALs.

\subsection{Pilot Variability Study of HS $1603+3820$}

As a pilot variability study of mini-BALs, we have been monitoring a $\mathrm{C}$ VI mini-BAL in the optically bright quasar $\mathrm{HS}$ $1603+3820\left(z_{\mathrm{em}}=2.542 ; \quad B=15.9\right)$ using primarily the high-dispersion spectrograph (HDS) on the Subaru Telescope (Misawa et al. 2003, 2005, 2007b). This mini-BAL spans a range of blueshifts of $8300-10,600 \mathrm{~km} \mathrm{~s}^{-1}$ relative to the quasar and shows both partial coverage and time variability. Our monitoring campaign spans more than four years (corresponding to about one year in the quasar rest frame). No correlations are seen between the profile model parameters, except for a possible correlation between total equivalent width and the coverage fraction (see Figure 5 of Misawa et al. 2007b). As argued by Misawa et al. (2007b), simple changes of ionization state or motion of a homogeneous gas parcel cannot reproduce the observed variations. The former requires much faster variations of the UV continuum flux than one would expect from such a luminous quasar (e.g., Giveon et al. 1999; Hawkins 2001; Kaspi et al. 2007). The latter requires that all the absorbing parcels of gas (that correspond to each of the absorption troughs) are at the same distance from the continuum source and cross our cylinder of sight at the same time and without discernible changes in their line-of-sight velocities, which is rather unlikely. Only two plausible scenarios remain for the origin of the variability: (1) continuum photons, redirected by a variable scattering medium into our cylinder of sight,
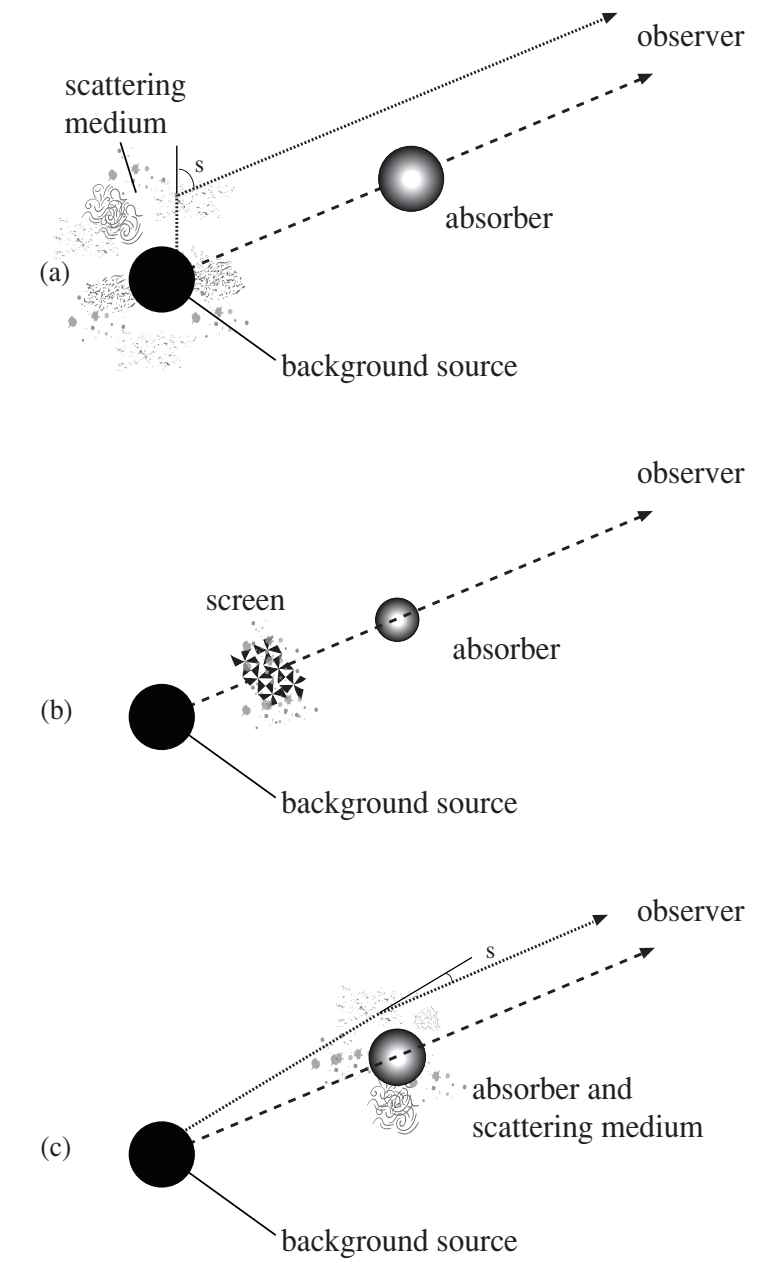

Figure 1. Three possible geometries that could lead to apparent partial coverage and variability as seen in the C VI mini-BAL in HS 1603+3820. The dashed lines represent the direct lines of sight toward the continuum source at the center of the accretion disk, whereas the dotted lines represent the paths of scattered photons. (a) The absorbing parcel of gas is larger than the background continuum source but photons are redirected around it via scattering in a corona surrounding the central continuum source. (b) The absorbing parcel of gas is small enough that it only occults a portion of the background continuum source. A variable, porous/ clumpy shield (e.g., the warm absorber) that modulates the transmitted flux is located between the $\mathrm{C}$ VI absorber and the continuum source. (c) Similar model to (a), but the scattering medium is surrounding the absorber rather than the continuum region. The scattering media in (a) and (c) are neither homogenous nor spherically symmetric about the continuum source or the absorber. The polarization degree depends on the scattering angle (denoted by $s$ ).

dilute the mini-BAL absorption and modulate its equivalent width according to the (variable) intensity of the scattered light (depicted in Figure 1(a)) and (2) rapid continuum fluctuations, coupled with coverage fraction fluctuations caused by a porous/ clumpy screen of variable optical depth located between the continuum source and the mini-BAL gas (Figure 1(b)). A detailed discussion of these scenarios is presented in Misawa et al. (2007b). A third possibility, not discussed explicitly by Misawa et al. (2007b), is that the scattering medium surrounds the absorber itself, as depicted in Figure 1(c).

\subsection{Spectropolarimetric Test of the Scattering Hypothesis}

The scattering scenario for the variability of the C VI miniBAL in HS $1603+3820$ can be tested through spectropolarimetric observations. Polarimetry and spectropolarimetry of BAL and non-BAL QSOs show that the former are on average more 


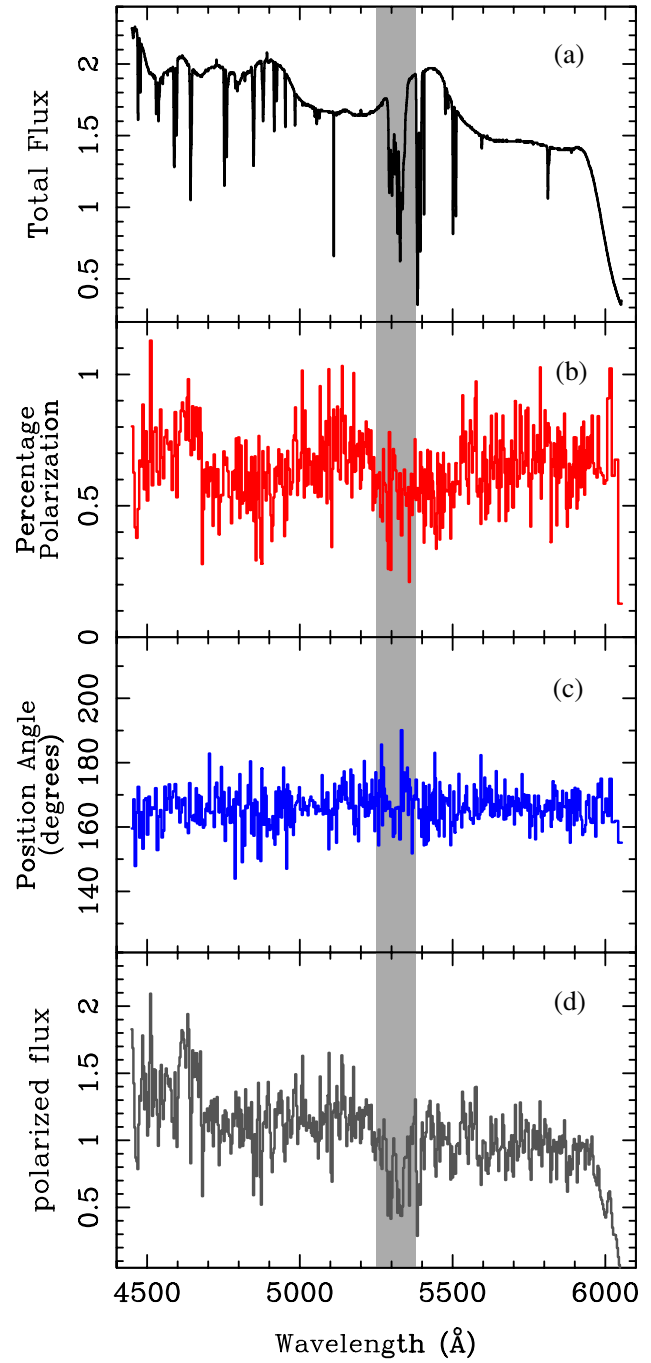

Figure 2. Summary of the results of the spectropolarimetric observations of HS $1603+3820$. (a) the total flux density spectrum $\left(f_{\lambda}\right.$ or $\left.f\right)$, (b) the percentage polarization spectrum $(p),(c)$ the polarization angle $(\theta$, in degrees), and (d) the polarized flux (the product of the fractional polarization and total light spectrum, $f \times p$ ). The fluxes are in units of $10^{-15} \mathrm{erg} \mathrm{cm}^{-2} \mathrm{~s}^{-1} \AA^{-1}$. The wavelength scale is in the frame of the observer. The shaded band shows the spectral region immediately around the $\mathrm{C}$ VI mini-BAL.

(A color version of this figure is available in the online journal.)

polarized than the latter, in the continuum. However, the distribution of BAL polarization levels is broad, with values between $0 \%$ and $8 \%$ (or even higher in some cases). In contrast, nonBAL QSOs have polarization levels less than $2.5 \%$ (e.g., Ogle et al. 1999; Schmidt \& Hines 1999; see, e.g., Figure 1 in the latter paper). Of more interest here is the change of polarization level between the continuum and the BAL troughs. The implications of those observations form the basis for our observational test. The BAL troughs are generally significantly more polarized than the continuum. The degree of polarization in the BAL troughs can reach 12\%-15\% (e.g., Hines \& Wills 1995; Cohen et al. 1995; Brotherton et al. 1997; Ogle et al. 1999; Schmidt \& Hines 1999; Scoggins et al. 2004). There are however examples where the BAL troughs are actually unpolarized (see Brotherton et al. 1997, for a particularly interesting case).

Two families of explanations for the observed increase in the polarization level in BAL troughs have been discussed in the literature. (1) Large-angle scattering from a medium that is off the cylinder of sight to the background source. Thus the scattered photons, which are also unattenuated, make up a significant fraction of the residual light in the BAL troughs and increase the degree of polarization (e.g., Goodrich \& Miller 1995; Lamy \& Hutsemékers 2004). (2) Resonant scattering within the absorber itself can also produce polarization with a level as high as $15 \%$ in the trough (e.g., Lee \& Blandford 1997; Wang et al. 2007). The former scenario appears more promising, because of the observed anti-correlations the degree of polarization $(p)$ and the detachment index (DI), ${ }^{8}$ and between the spectropolarimetric index $(\mathrm{SI})^{9}$ and DI, which argue for the presence of a scattering medium in the polar direction (Hutsemékers et al. 1998; Lamy \& Hutsemékers 2004).

In this paper, we present the results of spectropolarimetry of the mini-BAL quasar, HS 1603+3820. Using our data, we search for variations in the degree of polarization across the mini-BAL trough. The detection of a strong polarization signal in the miniBAL trough would lead us to infer that (1) scattering of photons around the absorber contributes to the partial coverage and (2) the variability of the mini-BAL can be caused by fluctuations in the amount of scattered light, as depicted in Figure 1(a). Furthermore, we would also expect this fractional polarization to increase as the coverage fraction decreases.

In Section 2, we describe the observations and data reduction and in Section 3, we describe the spectropolarimetric properties of HS 1603+3820. In Section 4, we discuss the constraints placed by our observations on possible scattering mechanisms in HS $1603+3820$ and we evaluate the scenarios for the variability of the mini-BAL in light of the new data. We adopt $z_{\mathrm{em}}=2.542$ as the systemic redshift of the quasar, which was estimated from its narrow emission lines (Misawa et al. 2003).

\section{OBSERVATIONS AND DATA REDUCTION}

The spectropolarimetric observation for HS 1603+3820 was carried out with the Faint Object Camera And Spectrograph (FOCAS; Kashikawa et al. 2002) on the Subaru Telescope, on 2008 August 7 and 8 (UT). The detector is a mosaic of two $4 \mathrm{k} \times 2 \mathrm{k}$ MIT CCDs with $15 \mu \mathrm{m}$ pixels. All the observations were carried out through a polarimetric unit that consists of a rotating superachromatic half-wave plate and a quartz Wollaston prism. Since the circular polarization is negligible for QSOs, we measured only linear polarization. We used a 0.8 slit, the $600 \mathrm{~mm}^{-1}$ Volume-Phase Holographic grism $\left(600 \_520 \mathrm{~nm}\right)$, and the L600 order-sorting filter. This setting results in a resolving power of $R \sim 1500$. A segment of the spectropolarimetric observation consists of exposures at four wave-plate position angles: $0.0,45.0,22.5$, and 67.5 . The integration time of each exposure was $900 \mathrm{~s}$, and the total integration time was $8 \mathrm{hr}$. The position angle of the entrance slit was set to be $180^{\circ}$. We also obtained spectra of an unpolarized flux standard star (BD+28 4211; Oke 1990) and a strongly polarized star (HD 204827). Spectra of a halogen lamp and a thorium-argon lamp were also obtained for flat fielding and wavelength calibration, respectively. The observation log is presented in Table 1.

The data were reduced in a standard manner, using IRAF. ${ }^{10}$ We extracted the spectra of HS $1603+3820$ and the standard stars

\footnotetext{
8 The detachment index (DI) is the onset velocity of a BAL trough in units of the half-width of the corresponding broad emission line (Weymann et al. 1991).

9 The spectropolarimetric index (SI) is a measure of the strength of the absorption in the polarized flux, $f_{\lambda} \times p_{\lambda}$, relative to the absorption in the direct flux, $f_{\lambda}$ (Lamy \& Hutsemékers 2004).

10 Image Reduction and Analysis Facility (IRAF) is distributed by the National Optical Astronomy Observatory, which is operated by the Association of Universities for Research in Astronomy Inc., under corporative agreement with the National Science Foundation.
} 
Table 1

Log of Observations

\begin{tabular}{|c|c|c|c|c|c|c|c|c|c|}
\hline $\begin{array}{c}\text { Target } \\
(1)\end{array}$ & $\begin{array}{l}\text { R.A. }^{\mathrm{a}} \\
(\mathrm{h} \mathrm{m} \mathrm{s}) \\
(2)\end{array}$ & $\begin{array}{c}\text { Decl. }^{\mathrm{a}} \\
(\mathrm{d} \mathrm{m} \mathrm{s}) \\
(3)\end{array}$ & $\begin{array}{c}m_{V} \\
(\mathrm{mag}) \\
(4)\end{array}$ & $\begin{array}{l}\text { Date } \\
\text { (5) }\end{array}$ & $\begin{array}{c}\text { Exposure } \\
\text { (s) } \\
\text { (6) }\end{array}$ & $\begin{array}{c}\mathrm{S} / \mathrm{N}^{\mathrm{b}} \\
\left(\text { pixel }^{-1} \text { ) }\right. \\
(7)\end{array}$ & $\begin{array}{l}p^{c} \\
(\%) \\
(8) \\
\end{array}$ & $\begin{array}{c}\theta^{\mathrm{d}} \\
(\mathrm{deg}) \\
(9)\end{array}$ & $\begin{array}{l}\text { Note } \\
\text { (10) }\end{array}$ \\
\hline HS1603+3820 & 160455.4 & +381202 & 15.9 & 2008 Aug 7 and 8 & 28800 & 175 & $0.64 \pm 0.15$ & 166 & Quasar \\
\hline $\mathrm{BD}+284211$ & 215111.1 & +285152 & 10.5 & 2008 Aug 7 and 8 & 1080 & & & & Unpolarized star \\
\hline HD 204827 & 212857.8 & +584423 & 8.0 & 2008 Aug 8 & 24 & & & & Strongly polarized star \\
\hline
\end{tabular}

Notes.

${ }^{a}$ J2000. 0 coordinates.

b Typical S/N at the continuum level.

${ }^{c}$ Average percentage polarization and rms dispersion over the observed wavelength range.

${ }^{\mathrm{d}}$ Polarization angle in degree.

from a 4."0 aperture along the slit (i.e., 13 binned pixels). The instrumental polarization was corrected using the spectrum of the unpolarized standard star. The instrumental depolarization was not corrected because it has been found experimentally that it is less than a few percent of the total polarization degree. Flux calibration was performed using the spectrum of the unpolarized standard star. The polarization angle was calibrated using the data of the strongly polarized star.

The Galactic ISM is also responsible for partial linear polarization, and both the degree and the position angle of polarization show systematic variations with wavelength (e.g., Messinger et al. 1997), which are most naturally explained in terms of the presence of two or more different dust components (Serkowski et al. 1975; Coyne 1974). We estimate the Galactic interstellar polarization toward the direction of HS $1603+3820$ to be $p_{\text {ISM }} \leqslant 0.19 \%$, following the procedure below. The total Galactic H I column density was measured as $1.20 \times 10^{20} \mathrm{~cm}^{-2}$ by the Leiden/Argentine/Bonn (LAB) Survey (Kalberla et al. 2005). The Galactic neutral and molecular hydrogen column density can be conventionally converted to a color excess by $\left\langle N\left(\mathrm{H} \mathrm{I}+\mathrm{H}_{2}\right) / E(B-V)\right\rangle=5.8 \times 10^{21} \mathrm{~cm}^{-2} \mathrm{mag}^{-1}$ (Bohlin et al. 1978), which gives $E(B-V)=0.021$ mag. Finally, we estimate the maximum polarization degree $\left(p_{\max }\right)$ using the empirical relation $p_{\max }=9.0 E(B-V)=0.19 \%$ (Serkowski et al. 1975). The Galactic interstellar polarization estimated above is considerably smaller than the observed polarization ( $p \sim 0.64 \%$ ) and does not show any clear spectral dependence in either the degree of polarization or position angle as we describe in the following section. The small Galactic interstellar polarization toward the quasar is also confirmed using the polarization catalog of Galactic stars (Heiles 2000). Therefore, we did not correct for the Galactic interstellar polarization.

\section{RESULTS}

Our primary results are illustrated in Figures 2 and 3. In Figure 2, we plot as a function of wavelength the total flux density per unit wavelength $\left(f_{\lambda}\right.$, hereafter $\left.f\right)$, linear polarization degree $(p)$, polarization position angle $(\theta)$, and polarized flux $(f \times p)$. We have omitted error bars because the signal-to-noise ratio $(\mathrm{S} / \mathrm{N})$ is high enough even without binning. An expanded view of these spectra around the C VI mini-BAL feature (in the wavelength interval shaded in Figure 2) is also shown in Figure 3 with $1 \sigma$ error bars included. In Figure 4, we show the normalized total flux spectrum in the immediate vicinity of the C VI mini-BAL profile, compared to previously published, higher-resolution spectra.

We obtain an average degree of linear polarization of $\langle p\rangle \approx$ $0.64 \%$ with a dispersion $\sigma_{p} \approx 0.15 \%$ in the continuum, which

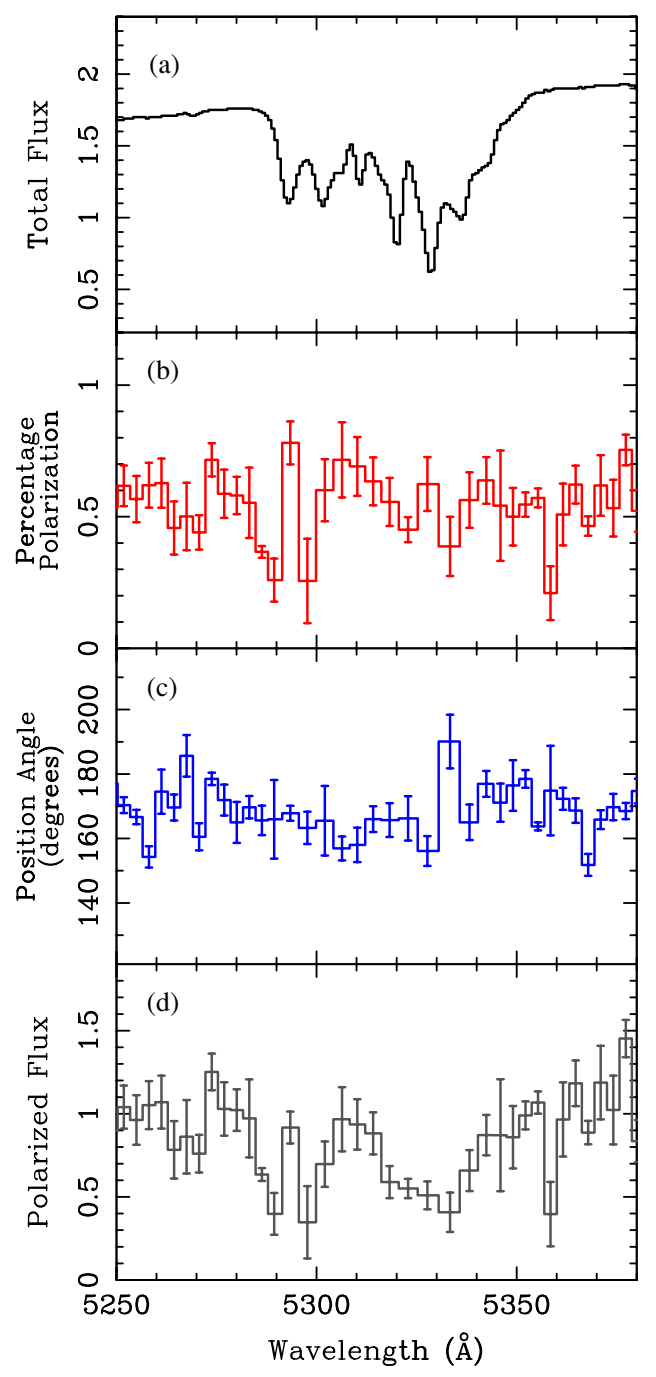

Figure 3. Expanded view of the spectra of Figure 2 in the vicinity of the $\mathrm{C}$ VI mini-BAL. The bin size is $\sim 0.8 \AA$ in (a) and $\sim 5 \AA$ in all other panels. In panels (b)-(d), we also plot " $1 \sigma$ " error bars.

(A color version of this figure is available in the online journal.)

is very similar to the average value in normal, non-BAL quasars. The polarization position angle is $\theta \approx 165^{\circ}$ over the entire range of the observed spectrum. The polarization level does not rise in the $\mathrm{C}$ VI mini-BAL trough, while it usually does in BAL profiles. We do not see any rotation of the polarization angle across the C VI mini-BAL feature, although such a rotation is observed in some BAL QSOs (e.g., Lamy \& Hutsemékers 2004). With 


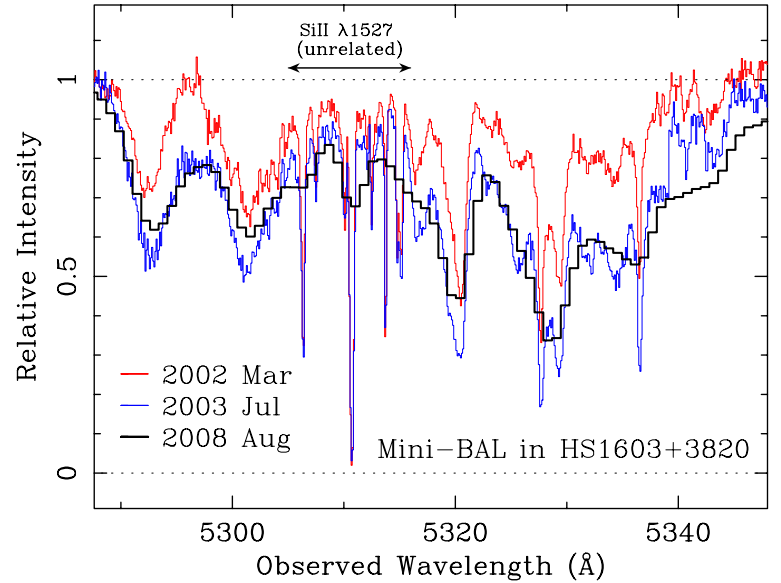

Figure 4. Normalized spectra of the quasar HS $1603+3820$ around the $\mathrm{C}$ VI mini-BAL for three epochs. The two higher-resolution spectra were taken with Subaru/HDS (Misawa et al. 2007b) and illustrate the full range of observed variability (the upper trace, in red, shows the spectrum from 2002 March, whereas the lower trace, in blue, shows the spectrum from 2003 July). The coarsely binned, thick black line shows the lower-resolution Subaru/FOCAS spectrum discussed in this paper (obtained in 2008 August). The narrower Si II lines are unrelated to the quasar and arising at a foreground galaxy.

(A color version of this figure is available in the online journal.)

the combination of high polarization sensitivity $(\delta p \sim 0.1 \%)$ and high resolution of our spectra, we would have detected any change in polarization degree or position angle rotation across the C VI mini-BAL trough of HS $1603+3820$, even if this were considerably smaller than what is typically observed in BAL quasars.

We also measure the following quantities from the spectrum: a detachment index of $\mathrm{DI} \approx 2.01$, a rest equivalent width of the red part of the $\mathrm{C}$ VI broad emission line of $\mathrm{C} \mathrm{VI}_{\mathrm{HREW}} \approx 5.5 \AA$, a logarithmic slope of the optical spectrum of $\alpha_{o} \approx 0.78$, a maximum offset velocity of the mini-BAL trough of $\Delta v_{\max } \approx$ $11,220 \mathrm{~km} \mathrm{~s}^{-1}$ (at the left edge of the bluest absorption component at $v_{\text {off }} \approx 10,600 \mathrm{~km} \mathrm{~s}^{-1}$ ), a spectropolarimetric index of $\mathrm{SI} \approx 1.18$, and a maximum polarization in the $\mathrm{C}$ VI mini-BAL trough of $p_{\max } \approx 0.82 \%$. A decrease in polarization is evident across all the emission lines in Figure 2(b), with the result that the polarized light spectrum plotted in Figure 2(d) shows no emission lines. The drop in polarization is most pronounced in the $\mathrm{Si}$ VI and $\mathrm{C}$ VI emission lines at approximately 4900 and $5400 \AA$. We measure a ratio of the polarization degree in the $\mathrm{C}$ VI emission line to that of the continuum adjacent to it of $p_{\text {C IV }} / p_{\text {cont }} \approx 0.8$. A similar behavior has been observed in some BAL QSOs (e.g., Ogle et al. 1999; Lamy \& Hutsemékers 2004).

\section{DISCUSSION}

An important result of our observations is that the degree of polarization does not change across the trough of the $\mathrm{C}$ VI mini-BAL in HS $1603+3820$. This result has direct implications for some of the scattering mechanisms that may operate in this object, as we discuss below. We specifically consider the following. (1) Large-angle electron scattering in a medium off the cylinder of sight and close to the background source, (2) electron scattering around the mini-BAL absorber, and (3) resonance scattering inside the absorber. We do not discuss scenarios involving dust scattering although we do note that they suffer from the following drawback: the grains must be close to the continuum source so as not to scatter a significant fraction of broad emission-line region photons, but then they will not be able to survive the intense radiation field at this distance.

1. Large-angle electron scattering around the background source. This is a promising mechanism, if the degree of polarization in the trough is large $(p \sim 10 \%)$, as often seen in spectra of BAL QSOs. A non-uniform, anisotropic "corona" around the continuum source is a candidate scattering medium (Figure 1(a)). In the case we are considering here, this mechanism could operate only to a small degree; i.e., the fraction of light that is scattered must be small so that it does not lead to a measurable increase in the polarization within the mini-BAL trough.

2. Electron scattering around the absorber. This mechanism is similar to the previous one, but the scattering medium surrounds the absorber, not the continuum source (Figure 1(c)). This corona could be the highly ionized atmosphere of the absorbing gas parcel. The degree of polarization depends on the absorber's size and distance from the continuum source, as well as its geometry. Thus, a filamentary absorber with an extended scattering corona, located very close to the source, is disfavored because in such a case the scattering angle (denoted by $s$ in Figure 1(c)) is large and the scattered photons will have a large polarization, in contradiction with our observations. On the other hand, if the absorber is very far from the background source (such that the extent of the scattering medium is much smaller than the distance from the background source), the scattering angle is inevitably small and so is the resulting polarization. Such a scenario remains viable in view of our results. In fact, the plausibility of this scenario is reinforced by the finding that the absorbers in some quasars are located at distances of several kiloparsec or more from the continuum source (Hamann et al. 2001; Korista et al. 2008; Dunn et al. 2010).

3. Resonance scattering inside the absorber. Resonance scattering of line photons within the absorber can produce some level of polarization. Lee et al. (1994) introduced this scenario, while Lee \& Blandford (1997) predicted a significant rise in the degree of polarization inside the absorption troughs. The predicted polarization levels in the absorption troughs are $p \sim 5 \%-15 \%$ depending on the geometry, which is in contradiction with our observations. Thus, we consider resonance scattering unlikely.

Considering the results of our spectropolarimetric observation, we can reject with some confidence the large-angle scattering scenario (Figure 1(a)) as the cause of partial coverage, hence the origin of variability of the C VI mini-BAL in HS $1603+3820$. The primary argument against this scenario is that the polarization level remains low in the mini-BAL trough and similar to the level out of the trough. The absorption depth of the $\mathrm{C}$ VI mini-BAL in the observation presented here is clearly smaller than the absorption depth in the 2003 July observation (see Figure 4). In the context of the scattering hypothesis, this suggests that there should be a considerable amount of scattered light in the absorption trough, yet we did not detect any change in polarization across the absorption trough. Similar arguments apply to the resonance scattering scenario. The only scattering scenario that remains viable is one in which the scattering medium surrounds the distant, intrinsic absorber (as depicted in Figure 1(c)). In this scenario, the scattering angle is small and so is the resulting degree of polarization. The observed coverage fraction sets an additional constraint on this scenario: the combination of scattering optical depth and solid angle in 
the scattering medium around the absorber must be such that it can redirect the appropriate number of photons toward the observer.

In view of the above, we favor the variable screen hypothesis for the variable mini-BAL observed in this object. This hypothesis is also supported by observations of X-ray spectral variability in other mini-BAL quasars, such as those reported by Ballo et al. (2008) and Giustini et al. (2010a, 2010b). Thus, X-ray observations of HS $1603+3820$ will be particularly useful since they can test this scenario for this particular quasar.

The research was supported by the Japan Society for the Promotion of Science through Grant-in-Aid for Scientific Research 21740150. T.M. acknowledges supports from the Special Postdoctoral Research Program of RIKEN and from the Sumitomo Foundation (090358). M.E. and J.C. acknowledge support from NSF grant AST-0807993. We thank the anonymous referee for a number of comments that helped us improve the paper.

\section{REFERENCES}

Ballo, L., Giustini, M., Schartel, N., Cappi, M., Jiménez-Bailón, E., Piconcelli, E., Santos-Lleó, M., \& Vignali, C. 2008, A\&A, 483, 137

Barlow, T. A., \& Sargent, W. L. W. 1997, AJ, 113, 136

Becker, R. H., et al. 2001, ApJS, 135, 227

Bohlin, R. C., Savage, B. D., \& Drake, J. F. 1978, ApJ, 224, 132

Brotherton, M. S., Tran, H. D., van Breugel, W., Dey, A., \& Antonucci, R. 1997, ApJ, 487, L113

Chartas, G. 2000, ApJ, 531, 81

Chartas, G., et al. 2009, New Astron. Rev., 53, 128

Churchill, C. W., Schneider, D. P., Schmidt, M., \& Gunn, J. E. 1999, AJ, 117, 2573

Cohen, M. H., Ogle, P. M., Tran, H. D., Vermeulen, R. C., Miller, J. S., Goodrich, R. W., \& Martel, A. R. 1995, ApJ, 448, L77

Coyne, G. V. 1974, AJ, 79, 565

de Kool, M., \& Begelman, M. C. 1995, ApJ, 455, 448

Dunn, J., et al. 2010, ApJ, 709, 611

Everett, J. E. 2005, ApJ, 631, 689

Ganguly, R., Eracleous, M., Charlton, J. C., \& Churchill, C. W. 1999, AJ, 117, 2594

Giustini, M., Cappi, M., Chartas, G., Eracleous, M., Vignali, C., \& Palumbo, G. G. C. 2010a, in ASP Conf. Ser., Accretion and Ejection in AGN: A global View, ed. L. Maraschi et al. (San Francisco, CA: ASP), in press (arXiv:0912.0024)

Giustini, M., Cappi, M., Chartas, G., Eracleous, M., Vignali, C., \& Palumbo, G. G. C. 2010b, in IAU Symp. 267, Co-evolution of Black Holes and Galaxies, ed. B. M. Peterson, R. Sommerville, \& T. Storchi-Bergmann (Cambridge: Cambridge Univ. Press), 397

Giveon, U., Maoz, D., Kaspi, S., Netzer, H., \& Smith, P. S. 1999, MNRAS, 306, 637

Goodrich, R. W., \& Miller, J. S. 1995, ApJ, 448, L73
Granato, G. L., De Zotti, G., Silva, L., Bressan, A., \& Danese, L. 2004, ApJ, 600,580

Gregg, M. D., Becker, R. H., \& de Vries, W. 2006, ApJ, 641, 210

Hamann, F., Barlow, T. A., \& Junkkarinen, V. 1997a, ApJ, 478, 87

Hamann, F., Barlow, T. A., Junkkarinen, V., \& Burbidge, E. M. 1997b, ApJ, 478,80

Hamann, F. W., Barlow, T. A., Chaffee, F. C., Foltz, C. B., \& Weymann, R. J. 2001, ApJ, 550, 142

Hawkins, M. R. S. 2001, ApJ, 553, L97

Heiles, C. 2000, AJ, 119, 923

Hewett, P. C., \& Foltz, C. B. 2003, AJ, 125, 1784

Hines, D. C., \& Wills, B. J. 1995, ApJ, 448, L69

Hutsemékers, D., Lamy, H., \& Remy, M. 1998, A\&A, 340, 371

Kalberla, P. M. W., Burton, W. B., Hartmann, D., Arnal, E. M., Bajaja, E., Morras, R., \& Pöppel, W. G. L. 2005, A\&A, 440, 775

Kashikawa, N., et al. 2002, PASJ, 54, 819

Kaspi, S., Brandt, W. N., Maoz, D., Netzer, H., Schneider, D. P., \& Shemmer, O. 2007, ApJ, 659, 997

Königl, A., \& Kartje, J. F. 1994, ApJ, 434, 446

Korista, K. T., Bautista, M. A., Arav, N., Moe, M., Costantini, E., \& Benn, C. 2008, ApJ, 688, 108

Lamy, H., \& Hutsemékers, D. 2004, A\&A, 427, 107

Lee, H.-W., \& Blandford, R. D. 1997, MNRAS, 288, 19

Lee, H.-W., Blandford, R. D., \& Western, L. 1994, MNRAS, 267, 303

Messinger, D. W., Whittet, D. C. B., \& Roberge, W. G. 1997, ApJ, 487, 314

Misawa, T., Charlton, J. C., Eracleous, M., Ganguly, R., Tytler, D., Kirkman, D., Suzuki, N., \& Lubin, D. 2007a, ApJS, 171, 1

Misawa, T., Eracleous, M., Charlton, J. C., \& Kashikawa, N. 2007b, ApJ, 660 , 152

Misawa, T., Eracleous, M., Charlton, J. C., \& Tajitsu, A. 2005, ApJ, 629, 115 (M05)

Misawa, T., Eracleous, M., Chartas, G., \& Charlton, J. C. 2008, ApJ, 677, 863

Misawa, T., Yamada, T., Takada-Hidai, M., Wang, Y., Kashikawa, N., Iye, M., \& Tanaka, I. 2003, AJ, 125, 1336

Murray, N., Chiang, J., Grossman, S. A., \& Voit, G. M. 1995, ApJ, 451, 498

Narayanan, D., Hamann, F., Barlow, T., Burbidge, E. M., Cohen, R. D., Junkkaribeb, V., \& Lyons, R. 2004, ApJ, 601, 715

Ogle, P. M., Cohen, M. H., Miller, J. S., Tran, H. D., Goodrich, R. W., \& Martel, A. R. 1999, ApJS, 125, 1

Oke, J. B. 1990, AJ, 99, 1621

Proga, D., Stone, J. M., \& Kallman, T. R. 2000, ApJ, 543, 686

Punsly, B. 1999, ApJ, 527, 624

Reichard, T. A., et al. 2003, AJ, 125, 171

Richards, G. T. 2001, ApJ, 133, 53

Richards, G. T., York, D. G., Yanny, B., Kollgaard, R. I., Laurent-Muehleisen, S. A., \& Vanden Berk, D. E. 1999, ApJ, 513, 576

Scannapieco, E., \& Oh, S. P. 2004, ApJ, 608, 62

Schmidt, G. D., \& Hines, D. C. 1999, ApJ, 512, 125

Scoggins, B., Brotherton, M. S., Becker, R. H., Tran, H. D., Gregg, M. D., White, R. L., \& Laurent-Muehleisen, S. 2004, BAAS, 36, 766

Serkowski, K., Mathewson, D. S., \& Ford, V. L. 1975, ApJ, 196, 261

Springel, V., Di Matteo, T., \& Hernquist, L. 2005, ApJ, 620, L79

Stocke, J. T., Morris, S. L., Weymann, R. J., \& Foltz, C. B. 1992, ApJ, 396, 487

Voit, G. M., Weymann, R. J., \& Korista, K. T. 1993, ApJ, 413, 95

Wang, H.-Y., Wang, T.-G., \& Wang, J.-X. 2007, ApJS, 168, 195

Weymann, R. J., Morris, S. L., Foltz, C. B., \& Hewett, P. C. 1991, ApJ, 373, 23

Wise, J. H., Eracleous, M., Charlton, J. C., \& Ganguly, R. 2004, ApJ, 613, 129 